\title{
Evidência de Validade entre o Inventário de Percepção de Suporte Familiar -IPSF e Familiograma -FG
}

\author{
Evidence of Validity between Perception of Family Support \\ Inventory and Familiogram $(F G)$
}

\author{
Makilim Nunes Baptista*, ${ }^{*}$, Maycoln Leôni Martins Teodoro ${ }^{b}$, Ricardo Vivian da Cunha ${ }^{b}$, \\ Priscilla Rodrigues $\operatorname{Santana}^{a}$, \& Adriana Munhoz Carneiro ${ }^{a}$ \\ ${ }^{a}$ Universidade São Francisco \& ${ }^{b}$ Universidade do Vale do Rio dos Sinos
}

\begin{abstract}
Resumo
A compreensão dos relacionamentos familiares está associada ao desenvolvimento de instrumentos psicológicos válidos e fidedignos. Neste sentido, o presente estudo pretendeu avaliar alguns indícios de validade do Inventário de Percepção de Suporte Familiar (IPSF) e do Familiograma (FG). Os dois instrumentos foram preenchidos por 260 universitários de duas cidades brasileiras. A amostra total foi composta por participantes de ambos os sexos, com idades entre 18 e 30 anos (Média=22,54, DP=3,69). Os resultados indicaram que os fatores Afetivo-Consistente e Adaptação Familiar possuem, em sua maioria, correlações moderadas com os construtos Afetividade e Conflito. Por outro lado, foram encontradas correlações baixas da Autonomia com os fatores do FG. Implicações destes resultados para a validade do IPSF e FG são discutidas.

Palavras-chave: Relações familiares; Validade de testes; Psicometria.

Abstract

The understanding of family relationships is related to the development of valid and reliable psychological instruments. In this way, the present study intended to evaluate some validity properties of the Perception of Family Support Inventory (PFSI) and Familiogram (FG). Both instruments were answered by 260 college students of two Brazilian cities. The total sample was composed by people of both genders, aged between 18 and 30 years old (Mean=22.54, SD=3.69). The results indicated that Emotional-Consistent and Family Adaptation factors moderately correlated with Affection and Conflict constructs. On the other hand, Autonomy has low correlations with FG factors. Implications of those results for the validity of PSFI and FG are discussed.

Keywords: Family relations; Validity of tests; Psychometrics.
\end{abstract}

A família atual possui uma organização na qual as relações internas como afeto, igualdade de direitos e carinho são valorizadas (Caldana, 1998; Ponciano \& Carneiro, 2003). Assim, a família consolida-se como um importante nicho para o desenvolvimento global do indivíduo, devido às influências que pode exercer em seu comportamento (Baptista, Baptista, \& Dias, 2001; Conte, 2001; Forman \& Forman, 1981). Os pais são considerados como a fonte primária que o ser humano possui de contato com o mundo, e desempenham um papel importante no comportamento e desenvolvimento da criança e adolescente, como mediadores entre eles e a sociedade, além de serem também aqueles que fornecem (ou que deveriam fornecer) todo tipo de suporte aos filhos (Baptista \& Oliveira, 2004; Rocha \& Brandão, 2001).

\footnotetext{
* Endereço para correspondência: Universidade São Francisco, Diretoria Acadêmica de Pós-Graduação, Rua Alexandre Rodrigues Barbosa, 45, Centro, Itatiba, SP, Brasil, CEP 13251-900. Tel.: (011) 4534 8040. E-mail: makilim.baptista@saofrancisco.edu.br
}

Este suporte é um fator importante tanto para o desenvolvimento global de crianças e adolescentes, quanto para a qualidade do relacionamento estabelecido com seus pais (Greenbergher, Chen, Tally, \& Dong, 2000). Esta afirmação tem como base a premissa de que é no contexto familiar que se podem aprender conceitos como amor, moral, ética, sociabilidade, dentre tantos outros. Porém, quando a família não proporciona um ambiente propício para o desenvolvimento da criança ou do adolescente, as chances deste indivíduo vir a desenvolver problemas de ordem psiquiátrica são maiores (Baptista et. al., 2001; Baptista \& Oliveira, 2004). Estudos sobre a família relatam o quanto esta pode influenciar a vida da criança e do adolescente tanto de forma positiva, quanto negativa (D'avila-Bacarji, Maturano, \& Elias, 2005; Greenbergher et al., 2000; Ivanova \& Israel, 2005; Montigny, Lacharite, \& Amyot, 2006; Pratta \& Santos, 2006).

Ivanova e Israel (2005) realizaram um estudo sobre a associação entre a estabilidade familiar e sintomas depressivos, cujos resultados também refletem a importância 
da família como fator que pode influenciar a aquisição de sintomatologia depressiva. Participaram da amostra coletada 158 estudantes, com idades entre 17 e 23 anos, de ambos os sexos. Foram aplicados quatro instrumentos, a saber, The Stability of Activities in the Family Environment (SAFE), que verificava a regularidade de atividades e/ou eventos referentes à rotina, como hora de dormir e de almoçar, o Beck Depression Inventory (BDI), o Attributional Style Questionnarie (ASQ), questionário composto de eventos hipotéticos negativos e positivos no qual os indivíduos devem atribuir causas às suas ocorrências, e o Life Events Checklist (LEC), que mede eventos estressantes entre adolescentes. Os resultados encontrados sugeriram que a estabilidade familiar protege os adolescentes com altas atribuições pessimistas de desenvolverem sintomas clinicamente significativos de depressão.

Já Baptista e Oliveira (2004) avaliaram a sintomatologia depressiva e o suporte familiar em 154 adolescentes provenientes de escola pública que cursavam o ensino médio. Os instrumentos foram um questionário de caracterização, cujo conteúdo baseava-se em fatores de risco para depressão, o Parental Bonding Instrument (PBI), que avalia carinho/indiferença e autonomia/superproteção dos pais e o Inventário de Depressão Infantil (CDI). Os resultados demonstraram que o suporte familiar esteve negativamente correlacionado com a sintomatologia depressiva, indicando que, quanto maior o grau da sintomatologia depressiva encontrada no adolescente, mais inadequada a forma do suporte familiar que é percebido por eles.

Elmaci (2006), procurando entender o mesmo construto, realizou uma pesquisa sobre papel do suporte em adolescentes, níveis depressivos e ajuste quando provindos de famílias inteiras e separadas. O objetivo do trabalho foi verificar se ocorria uma variação dos resultados de depressão e suporte em relação à situação das famílias. Participaram do estudo 203 adolescentes, sendo 101 provenientes de famílias separadas e 102 eram provenientes de famílias inteiras. Foram aplicados o Hacettepe Personality Inventory (HKE), que é utilizado de uma forma geral para determinar características de personalidade, o Beck Depression Inventory (BDI) e The Perceived Social Support Scale, utilizada para coletar dados dos estudantes sobre a percepção que possuíam sobre o suporte recebido e a constituição familiar. O autor encontrou como resultado uma diferença significativa entre os escores dos adolescentes de famílias separadas e nucleares em relação à percepção do suporte social recebido. Verificou-se também que os adolescentes provenientes de famílias separadas apresentavam-se com desajustes maiores, possuíam menor adaptabilidade a situações e níveis maiores de depressão do que os adolescentes provenientes de famílias inteiras.

Zdanowicz, Janne e Reynaert (2004) investigaram a correlação entre os tipos de relações familiares e a cren- ça que os adolescentes possuem sobre sua saúde, aplicando o Multidimensional Health Locus of Control (MHLC) e o The Family Adaptability and Cohesion Evaluation Scale II (FACES II) em 765 adolescentes. Posteriormente, este grupo foi comparado com um grupo de 358 adolescentes diagnosticados com desordens mentais. Em virtude dos resultados encontrados, que indicaram que a família tinha grande importância na constituição do senso de responsabilidade no adolescente por sua saúde, os autores concluíram que as relações familiares mostraram-se importantes para a aquisição de um controle do adolescente sobre seu bem-estar.

Com o mesmo intuito investigativo, Horta, Horta e Pinheiro (2006) realizaram uma pesquisa para avaliar a associação entre o consumo de drogas, como álcool, tabaco e drogas ilícitas em relação à presença e hábitos dos pais. Participaram da pesquisa 960 adolescentes com idades entre 15 e 18 anos, que responderam a um questionário que continha questões sobre comportamentos em saúde e uma entrevista. Como resultados, os autores verificaram que a presença física dos pais apareceu como um efeito protetor contra o uso de tabaco e, segundo os autores, provavelmente também contra as drogas. Outro resultado encontrado foi a presença da família como transmissora de comportamentos saudáveis que contribuem para a redução ou aumento do hábito de fumar. Assim, a partir dos dados discutidos, pode-se pressupor que o apoio e suporte proveniente da família seja um dos fatores que contribuem para o desenvolvimento saudável do indivíduo.

No contexto internacional pode-se observar um número expressivo de instrumentos que avaliam as relações familiares e parentais. Na maioria das vezes esses instrumentos já possuem diversas evidências de validade e são freqüentemente utilizados em pesquisas e/ou no cenário clínico (Bray, 1995). No Brasil, no entanto, percebe-se carência de instrumentos psicológicos em diversas áreas, inclusive na área de avaliação familiar. A seguir serão enfocados dois instrumentos que avaliam relações familiares e que vêm sendo estudados em termos de evidências de validade no Brasil, denominados Inventário de Percepção de Suporte Familiar -IPSF (Baptista, 2005, 2007) e Familiograma -FG (Teodoro, 2005, 2006).

O IPSF possui diversos estudos psicométricos de validade e fidedignidade. Dentre as evidências de validade destacam-se a de construto (Baptista, 2005, 2007) e as baseadas na relação com outras variáveis, tais como traços de personalidade, suporte social, autoconceito, saúde geral/mental, lócus de controle, auto-eficácia e vulnerabilidade ao estresse no trabalho (Aquino, 2007; Baptista, Alves, \& Santos, in press; Baptista \& Dias, 2007; Rigotto, 2006; Santos, 2006; Souza, 2007).

Estudos com o Familiograma (Teodoro, 2005; Teodoro \& Käppler, 2003) investigaram a relação da coesão familiar com o construto de qualidade de vida em crianças brasileiras de classe média. Os resultados mostraram que 
a coesão fraterna explicou cerca de $20 \%$ da variância da qualidade de vida destas crianças. Estes resultados confirmam pesquisas anteriores (Dundas, 1994; Phillips, West, Shen, \& Zheng, 1998) e apontam para a importância do sistema familiar na qualidade de vida de crianças. Reformulações na estrutura original do Familiograma foram apresentadas em Teodoro (2006). Neste estudo, foram investigadas algumas propriedades psicométricas da Afetividade e do Conflito em díades familiares. Os resultados apontaram para uma estrutura bi-fatorial com boa consistência interna.

Considerando os estudos acima relatados, percebe-se a importância de busca de evidências de validade para os instrumentos psicológicos. Além disso, nota-se a importância de busca de evidências de validade para os instrumentos psicológicos que avaliem este construto. Tendo em vista a resolução do Conselho Federal de Psicologia (CFP) n ${ }^{\circ}$ 25/2001 na qual determina para comercialização de um teste um conjunto de provas, realizadas por meio de parâmetros psicométricos adequados, que atestem sua qualidade e eficácia no objetivo proposto, o estudo de validade reveste-se de significante importância. De acordo com a resolução, a validade de um teste tem a função de verificar se o teste realmente mede o que se propõe, e quão bem o faz, permitindo ao avaliador a interpretação dos escores obtidos no teste aplicado.

Neste sentido, o estudo de validade encontra-se entre um destes parâmetros, e é de significante importância. É por meio do acumulo de evidências de validade que se determina o grau em que sua interpretação e utilização se adéquam aos conceitos psicológicos que dão base a investigação proposta (American Educational Research Association, American Psychological Association, \& Nacional Council on Measurement in Education [AERA, APA, \& NCME], 1999; Anastasi \& Urbina, 2000; Urbina, 2007). Existem diferentes formas de se verificar a validade de um teste, e uma delas é a busca de evidências de validade com relação a um critério. Dentro desta categoria existem várias formas de se conseguir tal evidência, inclusive a correlação com outros testes. Tal método pode ser realizado com um teste já padronizado e com evidências de validade, que meça um construto semelhante ou que seja próximo ao teste que está sendo estudado; ou pode ser realizado com um teste que mensure um construto oposto. A esse tipo de evidência de validade dá-se o nome de validade convergente-discriminante (AERA, APA, \& NCME, 1999, Urbina, 2007). De acordo com padrões nacionais (CFP, 2003) e internacionais (Prieto \& Muñiz, 2000), é desejável que um teste para ser utilizado em psicodiagnósticos apresente uma amostra de tamanho moderado $(200<N<500)$ a grande $(N>=500)$ e uma correlação convergente variando de ade-quada com alguma carência $(0,25 \leq 0,40)$; adequada $(0,40 \leq 0,50)$; boa $(0,50 \leq 0,60)$ e excelente (acima de 0,60$)$.

O presente estudo descreve os resultados do Inventário de Percepção de Suporte Familiar e do Familiograma em uma amostra de universitários e investiga alguns indícios de validade convergente entre os dois instrumentos, esperando-se encontrar correlações adequadas entre as dimensões dos instrumentos. Além disso, esta pesquisa busca comparar a intensidade do suporte familiar entre grupos de famílias classificadas de acordo com a combinação dos construtos afetividade e conflito.

\section{Método}

\section{Participantes}

Participaram deste estudo 260 universitários, sendo 116 do interior do estado de São Paulo e 144 do Rio Grande do Sul. A amostra total foi composta por 73 participantes do sexo masculino $(28,10 \%)$ e 187 do sexo feminino $(71,90 \%)$. A idade variou de 18 a 30 anos (Média=22,54, $D P=3,69)$. Com relação ao local de moradia do participante, constatou-se que 171 moravam com os pais $(65,70 \%)$.

Não houve diferença significativa quando comparadas a média de idade $(t=1,29, \mathrm{~ns})$ e sexo $\left(\chi^{2}=0,80, \mathrm{~ns}\right)$ do grupo de participantes de São Paulo e Rio Grande do Sul. Somente com relação ao local de moradia do estudante foi encontrada, dentro do grupo de São Paulo, uma maior freqüência na categoria "morar sem os pais" $\left(\chi^{2}=7,34, p<0,01\right)$. Levando-se em conta o caráter de conveniência da amostra e que a mesma provém de duas universidades, não se pode considerar esta como representativa de estudantes universitários brasileiros.

\section{Procedimentos}

O contato com os universitários foi feito por meio de visitas à sala de aula dentro das universidades. Os pesquisadores explicavam o conteúdo do estudo e deixavam claro que os dados pessoais seriam mantidos em sigilo. Os participantes poderiam deixar qualquer pergunta em branco ou se retirar da pesquisa assim que desejassem. O projeto foi aprovado pelo comitê de ética em uma das universidades dos autores (Processo 06/32). A aplicação dos testes foi feita coletivamente e durou aproximadamente 30 minutos.

\section{Instrumentos}

Inventário de Percepção de Suporte Familiar ([IPSF]; Baptista, 2005, 2007). O IPSF é um inventário composto por 42 itens divididos em três fatores. O primeiro fator denominado Afetivo-Consistente possui 21 itens a avalia a expressão de afetividade entre os membros familiares (verbal e não verbal), o interesse, proximidade, acolhimento, comunicação, interação, respeito, empatia, clareza nas regras intrafamiliares, consistência de comportamentos e verbalizações e habilidades na resolução de problemas. O fator denominado de Adaptação Familiar ficou composto por 13 itens, sendo expresso por perguntas referentes a sentimentos e comportamentos negativos em relação à família, tais como 
raiva, isolamento, incompreensão, exclusão, não pertencimento, vergonha, irritação, relações agressivas (brigas e gritos), além de percepção de que os familiares competem entre si, são interesseiros e se culpam nos conflitos, ao invés de tentarem inter-relações mais pró-ativas. Esta dimensão foi pontuada inversamente, logo, como ocorre com os outros fatores, quanto maior a pontuação, mais adequado é o suporte familiar, o que significa mais comportamentos positivos em relação à família (pouca ou nenhuma raiva, isolamento, sentimentos de incompreensão, exclusão, vergonha, irritação, relações agressivas, etc). Por último, o fator denominado de Autonomia foi composto por 8 itens e possui questões que podem assinalar relações de confiança, liberdade e privacidade entre os membros. Os índices de confiabilidade, expressos pelo Alfa de Cronbach, demonstraram que o fator 1 apresentou o Alfa de 0,91 , o fator 2 de 0,90 e, o fator 3 de 0,78 , além do valor de 0,93 para a escala total. O IPSF é respondido por intermédio de uma escala Likert de três pontos, pontuando-se 2 para respostas "sempre"; 1 para "às vezes" e 0 para "nunca" (as pontuações gerais variam de 0 a 84). No presente estudo, foi encontrado um índice Alfa de 0,91 para o fator I (Afetivo-consistente), 0,84 para o fator II (Adaptação Familiar) e 0,77 para o fator III (Autonomia). O somatório total do inventário apresentou um Alfa de Cronbach de 0,93. Os resultados mostraram que o instrumento manteve índices adequados de consistência interna na amostra investigada.

Familiograma (Teodoro, 2005, 2006). O Familiograma avalia a percepção da afetividade e o conflito familiar nas díades familiares (por exemplo, pai-mãe, filho-pai, filho-mãe etc.). Afetividade é definida como um conjunto de emoções positivas existentes no relacionamento interpessoal. Já conflito é caracterizado como uma gama de sentimentos negativos que podem ser tanto uma fonte geradora de estresse como de agressividade dentro do sistema familiar. Para esta pesquisa, investigaram-se as díades Filho-Pai, Filho-Mãe e Pai-Mãe na perspectiva do filho. Além destas díades, foi calculado um escore familiar total para afetividade e outro para conflito que consiste no escore médio obtido para as três díades estudadas. Para cada uma destas díades, o participante tinha que informar, por meio de uma lista de adjetivo e uma escala Likert variando de um a cinco, como era cada relacionamento. Para o construto afetividade, foram usados os seguintes adjetivos: carinhoso, alegre, agradável, verdadeiro, afetivo, protetor, amoroso, acolhedor, harmonioso, atencioso, precioso. Já o conflito foi avaliado por meio dos seguintes adjetivos: confuso, nervoso, estressante, baixo-astral, ruim, sufocante, tenso, frio, difícil, agressivo, chato. A pontuação do Familiograma varia de 11 a 55 para cada construto, sendo que quanto maior for o escore, maior será a percepção de afetividade e conflito. O Familiograma permite a classificação das famílias em quatro diferentes categorias de acordo com a intensidade da afetividade e do conflito familiar. As famílias pertencentes ao Tipo I são aquelas descritas como tendo alta afetividade e baixo conflito. Famílias do Tipo II possuem alta afetividade e alto conflito. Já as famílias classificadas como Tipo III possuem baixa afetividade e baixo conflito, enquanto as do Tipo IV possuem baixa afetividade e alto conflito.

Teodoro (2006) demonstrou a existência de uma estrutura bi-fatorial e Alfas de Cronbach variando de 0,87 até 0,97 para o Familiograma. No presente estudo, as análises de consistência interna foram de e 0,96 (Afetividade) e 0,94 (Conflito) para a díade participante-pai, 0,95 (Afetividade) e 0,90 (Conflito) para a díade participante-mãe e 0,97 (Afetividade) e 0,93 (Conflito) para a díade Pai-Mãe, indicando que o instrumento manteve a consistência interna na amostra estudada.

\section{Análise dos Dados}

A investigação da consistência interna dos dados do Inventário de Percepção de Suporte Familiar (IPSF) e do Familiograma foi feita com o Alfa de Cronbach. Para as comparações entre o sexo e idade dos participantes utilizou-se o teste $t$ de Student para amostras independentes e a correlação de Pearson. Para as comparações entre os escores do IPSF e o Familiograma foram utilizadas as correlações de Pearson. A comparação da intensidade de suporte familiar em cada tipo de família de acordo com o FG foi feita com a Análise de Variância One-way e teste pos hoc de Bonferroni. Toda a análise estatística foi realizada no programa estatístico SPSS 15.0.

\section{Resultados}

O presente trabalho investigou variáveis familiares como o suporte, a afetividade e o conflito em uma amostra de universitários. Além disso, explorou algumas propriedades psicométricas de dois instrumentos que avaliam as relações familiares. A apresentação dos resultados será dividida em três seções. A primeira apresentará os resultados descritivos e as análises relacionadas ao sexo, idade e local de moradia do participante. A segunda parte descreverá os dados relacionados à validade convergente entre o IPSF e Familiograma. Finalmente, a última seção conterá uma comparação entre tipos de famílias formadas a partir da combinação dos construtos afetividade e conflito (Familiograma) e o suporte familiar recebido (IPSF).

A Tabela 1 apresenta os resultados descritivos do Inventário de Percepção de Suporte Familiar e do Familiograma. Análises comparativas de médias com o teste $t$ de Student não indicaram nenhuma diferença significativa entre os grupos Masculino e Feminino. No intuito de investigar a relação entre os escores dos instrumentos e a idade do participante, foram feitas análises de correlação de Pearson. Entre todas as relações analisadas, foi encontrada somente uma correlação significativa negativa entre a intensidade do conflito Filho-Pai e a idade 
Tabela 1

Resultados Descritivos do Inventário de Percepção do Suporte Familiar (IPSF) e do Familiograma (FG) (n=260)

\begin{tabular}{|c|c|c|c|c|c|c|}
\hline Instrumento & Fator & Média $(D P)$ & Mediana & $1^{\circ}$ Quartil & $3^{\circ}$ Quartil & Min/Máx \\
\hline \multirow[t]{4}{*}{ IPSF } & Afetivo Consistente & $27,76(8,43)$ & 29,00 & 23,00 & 34,00 & $2-42$ \\
\hline & Adaptação Familiar & $4,89(4,06)$ & 4,00 & 2,00 & 7,00 & $0-19$ \\
\hline & Autonomia & $13,08(2,74)$ & 14,00 & 11,25 & 15,00 & $1-16$ \\
\hline & Total & $62,12(12,25)$ & 64,00 & 55,00 & 71,00 & $17-83$ \\
\hline \multirow[t]{4}{*}{ FGAfetividade } & Filho-Mãe & $45,45(7,91)$ & 47,00 & 41,00 & 52,00 & $17-55$ \\
\hline & Filho-Pai & $40,62(11,24)$ & 44,00 & 32,75 & 49,00 & $11-55$ \\
\hline & Pai-Mãe & $38,59(11,94)$ & 41,00 & 32,00 & 47,00 & $11-55$ \\
\hline & Familiar & $41,58(8,54)$ & 43,00 & 35,67 & 47,33 & $13-55$ \\
\hline \multirow[t]{4}{*}{ FGConflito } & Filho-Mãe & $18,34(6,86)$ & 16,00 & 13,00 & 23,00 & $11-47$ \\
\hline & Filho-Pai & $19,72(9,03)$ & 17,00 & 12,00 & 25,00 & $11-50$ \\
\hline & Pai-Mãe & $22,02(9,49)$ & 20,00 & 14,75 & 28,00 & $11-55$ \\
\hline & Familiar & $20,11(6,89)$ & 18,67 & 14,33 & 25,33 & $11-38$ \\
\hline
\end{tabular}

Tabela 2

Escores de Correlação de Pearson entre o Inventário de Percepção de Suporte Familiar (IPSF) e o Familiograma $(F G)(n=260)$

\begin{tabular}{lcccc}
\hline & Afetivo Consistente & Adaptação Familiar & Autonomia & Total IPSF \\
\hline Afetividade (Filho-Mãe) & $0,56 * * *$ & $0,47 * * *$ & $0,24 * * *$ & $0,57 * * *$ \\
Afetividade (Filho-Pai) & $0,52 * * *$ & $0,33 * * *$ & $0,18 * *$ & $0,48 * * *$ \\
Afetividade (Pai-Mãe) & $0,48 * * *$ & $0,38 * * *$ & $0,16 * *$ & $0,48 * * *$ \\
Afetividade Familiar & $0,59 * * *$ & $0,45 * * *$ & $0,24 * * *$ & $0,58 * * *$ \\
Conflito (Filho-Mãe) & $-0,30 * * *$ & $-0,40 * * *$ & $-0,20 * * *$ & $-0,37 * * *$ \\
Conflito (Filho-Pai) & $-0,38 * * *$ & $-0,37 * * *$ & $-0,21 * * *$ & $-0,44 * * *$ \\
Conflito (Pai-Mãe) & $-0,45 * * *$ & $-0,44 * * *$ & $-0,14 *$ & $-0,50 * * *$ \\
Conflito Familiar & $-0,48 * * *$ & $-0,50 * * *$ & $-0,23 * * *$ & $-0,55 * * *$ \\
\hline
\end{tabular}

Nota. $* * * p<0,001, * * p<0,01, * p<0,05$.

( $r=-0,17, p<0,01)$. Com relação ao local de moradia (morando ou não com os pais), não foi encontrada nenhuma diferença significativa entre os grupos.

As análises de validade convergente entre o IPSF e o Familiograma foram feitas por meio dos escores de correlação de Pearson. Os resultados das correlações estão descritos na Tabela 2 .

De modo geral, foram encontradas correlações significativas variando de fracas (autonomia com as outras dimensões) a moderadas em todas as relações investigadas. Com relação à dimensão Afetivo-consistente do IPSF, pode-se observar na Tabela 2 correlações que variam de 0,48 a 0,59 com o construto afetividade do Familiograma. Já com o construto conflito, o fator afetivo consistente possui correlações negativas variando de adequadas com carências a adequadas $(-0,30$ a -0,48). $O$ fator 2 do IPSF (adaptação familiar) correlacionou-se positivamente (variação de 0,33 a 0,47 ) com a afetividade, variando de adequadas com carências a adequadas, e negativamente com o conflito $(-0,37$ a $-0,50)$, também variando de adequadas com carência a adequadas. Finalmente, o terceiro fator do IPSF (autonomia) apresentou correlações ina- dequadas e positivas com a afetividade $(0,16$ a 0,24$)$ e inadequadas e negativas com o conflito $(-0,14$ a -0,23).

Quando retiradas as correlações entre a dimensão de autonomia do IPSF com o Familiograma, já que o FG não se propõe a avaliar este construto, observou-se que, dentre todas as 24 correlações possíveis, o panorama muda substancialmente. Sendo assim, 25,0\% $(n=6)$ das correlações podem ser consideradas adequadas com alguma carência, 41,6\% $(n=10)$ das correlações como adequadas e, 33,4\% $(n=8)$ como sendo correlações aceitáveis pelos parâmetros internacionais e nacionais.

No intuito de comparar os escores de suporte familiar do IPSF com tipos de família advindos da combinação dos construtos afetividade e conflito do Familiograma, procedeu-se a uma classificação como descrita anteriormente. Para a classificação em afetividade e conflito baixo e alto, utilizou-se a mediana descrita na Tabela 1. Utilizando-se esta classificação, obteve-se 39,5\% de famílias classificadas como Tipo I, 9,0\% de Tipo II, 12,4\% como Tipo III e 39,0\% no Tipo IV.

Os escores de suporte familiar total e de cada fator do IPSF foram comparados entre os grupos de famílias 
Baptista, M. N., Teodoro, M. L. M., Cunha, R. V., Santana, P. R. \& Carneiro, A. M. (2009). Evidência de Validade entre o Inventário de Percepção de Suporte Familiar - IPSF e Familiograma - FG.

categorizadas por meio de análise de variância One-way e pos hoc Bonferroni. Análise de variância com a soma do IPSF mostrou resultado significativo $(F=25,33$, $p<0,001)$. Testes pos hoc indicaram que o grupo classificado como Tipo IV (baixa afetividade e alto conflito) possui significativamente menos suporte familiar do que todos os outros grupos. Análises de variância com os fatores do IPSF indicaram diferenças significativas para o fator afetivo consistente $(F=25,98, p<0,001)$, para a adaptação familiar $(F=15,38, p<0,001)$ e para a autonomia $(F=6,56, p<0,001)$. Análises pos hoc para o fator I indicaram que famílias do Tipo I (alta afetividade e baixo conflito) pontuam mais no construto afetivo consistente do que as famílias do Tipo III (baixa afetividade e baixo conflito) e IV (baixa afetividade e alto conflito). Já as famílias do Tipo II possuem significativamente mais o suporte afetivo do que as famílias do Tipo IV. Análises de Bonferroni para o fator II do IPSF indicaram que as famílias do Tipo IV (baixa afetividade e alto conflito) pos-suem uma adaptação familiar menos adequada do que todos os outros tipos. Já para o fator III, análises indicaram que as famílias o Tipo I (alta afetividade e baixo conflito) possuem mais autonomia do que as do Tipo II e IV.

\section{Discussão e Considerações Finais}

A investigação dos relacionamentos entre os membros da família desempenha um importante papel na compreensão do funcionamento familiar. Tendo em vista a importância do aspecto cultural na estruturação e definição das relações familiares, fazem-se necessárias pesquisas que foquem tanto o desenvolvimento quanto a investigação das propriedades psicométricas de instrumentos que estejam adaptados para as necessidades locais. Neste sentido, o presente estudo teve como principal objetivo avaliar algumas propriedades psicométricas do Inventário de Percepção de Suporte Familiar (IPSF) e do Familiograma (FG). Os resultados foram comparados no intuito de buscar evidências de validade entre ambos os instrumentos.

Análises comparativas entre os grupos masculino e feminino não demonstraram diferença significativa quanto aos construtos de suporte familiar, afetividade e conflito. Com relação à idade, foi encontrada uma análise significante em doze realizadas. Tendo em vista esta proporção, é bem provável que esta correlação esteja vinculada mais a peculiaridades da amostra investigada e ao erro Tipo I do que a um perfil que possa ser encontrado em outros estudos regularmente. Algumas pesquisas que investigaram as relações familiares em grupos de crianças e adolescentes encontraram algumas diferenças significativas, como uma menor coesão nos participantes mais velhos (Coakley, Holmbeck, Friedman, Greenley, \& Thill, 2002; Feldman \& Gehring, 1988). Esta diferença foi explicada pela mudança ocorrida durante a fase de transição para adolescência e nas negociações e novas definições de papéis dentro da família (Larson, Richards, Moneta, Holmbeck, \& Duckett, 1996; Steinberg, 1990). A ausência de um padrão nas correlações dos instrumentos com a idade nesta amostra (adultos jovens) sugere que estes padrões já estejam estabilizados nesta fase da vida adulta.

Os indícios de validade convergente entre o IPSF e o Familiograma foram apresentados na Tabela 2. As correlações mais intensas foram encontradas entre os fatores Afetivo-consistente (IPSF) e Afetividade (FG) (variação de 0,48 a 0,59 ). A magnitude deste resultado pode ser explicada pela proximidade teórica dos construtos, que avaliam aspectos afetivos do relacionamento familiar. Da mesma forma, foram encontradas correlações significativas (variando entre 0,33 a 0,47 ) ente o fator Adaptação familiar (IPSF) e a Afetividade.

Com relação ao Conflito (FG), os coeficientes de Pearson variaram de $-0,30$ a $-0,48$ com o construto Afetivo Consistente e de -0,37 a -0,50 com a Adaptação Familiar (IPSF). Estes resultados apontam para indícios de validade divergente entre a percepção de conflito no sistema familiar e os sentimentos de suporte afetivo e adaptação familiar.

O fator Autonomia (IPSF) foi o que apresentou os menores escores de correlação com as dimensões do Familiograma. Os escores deste construto com a Afetividade variaram de 0,16 a 0,24 , enquanto que, com o Conflito, ficaram de $-0,14$ a $-0,23$. A presença de correlações fracas indicando ortogonalidade entre a autonomia e os construtos do Familiograma era esperada, na medida em que já existe, teoricamente, uma diferenciação entre a idéia de autonomia e as relações familiares (Cluff, Hicks, \& Madsen, 1994). Deste modo, os resultados do presente estudo confirmam a idéia destes autores, na qual a presença de comportamentos que demonstrem independência e autonomia com relação ao sistema familiar não implica a ausência de vínculo emocional.

Um resumo dos resultados sugere que as correlações encontradas apontam para indícios de validade convergente entre os fatores Afetivo-consistente e Adaptação familiar (IPSF) com os construtos Afetividade e Conflito (Familiograma), uma vez que de acordo com os padrões nacionais (CFP, 2003) e internacionais (Prieto \& Muñiz, 2000) de validação de testes psicológicos, espera-se que a correlação entre dois testes, que meçam construtos relacionados, apresentem correlações acima de 0,25. Já com relação ao fator Autonomia (IPSF), foram encontradas correlações inadequadas, sugerindo maior independência com relação aos construtos do Familiograma. Destaca-se que, quando são retirados os resultados do fator Autonomia, todas as 24 correlações restantes enquadram-se em categorias que variam de adequadas com alguma carência a boas, segundo a definição de Prieto e Muñiz (2000), o que reforça os indícios de validade convergente entre o IPSF e Familiograma. 
A análise dos resultados apresentados na Tabela 2 deve ser feita levando-se em consideração a existência de diferenças metodológicas e conceituais entre os dois instrumentos. Enquanto o IPSF utiliza uma idéia geral de família em que não são fornecidos os papéis dos membros pelo investigador, o Familiograma avalia as díades familiares. No presente estudo, as díades estudadas foram limitadas às formadas pelo filho, pai e mãe. Esta diferença metodológica pode gerar concepções de família diferentes, influenciado assim, a intensidade das correlações pesquisadas. No entanto, mesmo com as possíveis discrepâncias na concepção de família dos dois instrumentos, os resultados de validade convergente foram satisfatórios.

Com o intuito de comparar a intensidade do suporte familiar em famílias classificadas como tendo Afetividade baixa/alta e Conflito baixo/alto, foi elaborada uma classificação de tipos familiares (vide Quadro I). Esta classificação permite categorizar cada família em um determinado tipo, de acordo com a mediana dos construtos Afetividade e Conflito. Os resultados apontaram que famílias com baixa Afetividade e alto Conflito (Tipo IV) possuem menos suporte familiar do que as do Tipo I (alta Afetividade e baixo Conflito), II (alta Afetividade e alto conflito) e III (baixa Afetividade e baixo Conflito). A utilização desta tipologia pode ser útil na avaliação e identificação de famílias mais vulneráveis e que precisam de uma intervenção clínica.

As análises do presente estudo apontam, de modo geral, para a existência de validade convergente entre os fatores do IPSF e do Familiograma, levando-se em consideração que ambos os instrumentos possuem características em comum e outras individualizadas. Apesar de algumas limitações de amostragem, os resultados são importantes, na medida em que são poucos os instrumentos de avaliação familiar validados no Brasil. No entanto ressalta-se a necessidade de novos estudos que investiguem, principalmente, o desempenho de grupos clínicos nestes instrumentos.

É interessante notar que o clínico ou pesquisador interessado em avaliar a questão familiar poderá escolher utilizar um ou outro instrumento, dependendo de seus objetivos, escolhas teóricas e/ou avaliação psicométrica dos instrumentos. Por exemplo, se o intuito for avaliar as suposições que cada membro familiar tem do outro, o instrumento mais adequado pode ser o Familiograma, no entanto se o objetivo seja avaliar qual a percepção que o indivíduo tem do suporte que recebe, independente do membro que fornece tal suporte, o instrumento de opção pode ser o IPSF.

Além dessa questão, como aponta Bray (1995), os instrumentos que avaliam a família podem ser desenvolvidos empiricamente, embasados em teorias prévias ou serem construídos de forma combinada, o que também pode gerar diferenças em suas comparações e qualidades psicométricas distintas. De forma geral, é fundamental que dentro da área da avaliação psicológica haja, em primeiro lugar, instrumentos válidos e fidedignos para serem utilizados e, em segundo lugar, respeitada essa primeira premissa, quanto maior for a quantidade e variedade desses instrumentos em determinada área, maior a possibilidade de escolha adequada para os diversos objetivos propostos pelos avaliadores, sendo que a comunidade de pesquisadores e clínicos acabam tendo vantagens diversas.

\section{Referências}

American Educational Research Association., American Psychological Association., \& Nacional Council on Measurement in Education. (1999). Standards for educational and psychological testing. Washington, DC: American Educational Research Association.

Anastasi, A., \& Urbina, S. (2000). Fidedignidade. In A. Anastasi \& S. Urbina, Testagem Psicológica (pp. 54-106). Porto Alegre, RS: Artmed.

Aquino, R. R. (2007). Inventário de Percepção de Suporte Familiar (PSF) e Escala de Vulnerabilidade ao Estresse no Trabalho (EVENT): Evidência de Validade. Dissertação de Mestrado não-publicada, Universidade São Francisco, Itatiba, SP.

Baptista, M. N. (2005). Desenvolvimento do Inventário de Percepção de Suporte Familiar (IPSF): Estudos psicométricos preliminares. Psico-USF, 10, 11-19.

Baptista, M. N. (2007). Inventário de Percepção de Suporte Familiar (IPSF): Estudo componencial em duas configurações. Psicologia: Ciência e Profissão, 27, 496-509.

Baptista, M. N., Alves, G. A. S., \& Santos, T. M. M. (in press). Suporte familiar, auto- eficácia e lócus de controle: Evidências de validade entre os construtos. Psicologia: Ciência e Profissão.

Baptista, M. N., Baptista, A. S. N., \& Dias, R. R. (2001). Estrutura e suporte familiar como fatores de risco na depressão de adolescentes. Psicologia: Ciência e Profissão, 21, 52-61.

Baptista, M. N., \& Dias, R. R. (2007). Fidedignidade do Inventário de Percepção de Suporte Fatorial - IPSF. Avaliação Psicológica, 6, 33-37.

Baptista, M. N., \& Oliveira, A. A. (2004). Sintomatologia de depressão e suporte familiar em adolescentes: Um estudo de correlação. Revista Brasileira de Crescimento e Desenvolvimento Humano, 14, 58-67.

Bray, J. H. (1995). Family assessment: Current issues in evaluating families. Family Relations, 44, 469-477.

Caldana, R. H. L. (1998). A criança e sua educação na família no início do século: Autoridade, limites e cotidiano. Temas em Psicologia, 6, 87-103.

Cluff, R. B., Hicks, M. W., \& Madsen, C. H. (1994). Beyond the circumplex model: I. A moratorium on curvilinearity. Family Process, 33, 455-470.

Coakley, R. M., Holmbeck, G. N., Friedman, D., Greenley, R. N., \& Thill, A. W. (2002). A longitudinal study of pubertal timing, parent-child conflict, and cohesion in families of young adolescents with Spina Bifida. Journal of Pediatric Psychology, 27, 461-473.

Conselho Federal de Psicologia. (2001). Resolução CFP $n^{o}$ 25/2001. Retrieved August 13, 2007, from http:// www.crp07.org.br/upload/legislacao/legislacao46.pdf 
Baptista, M. N., Teodoro, M. L. M., Cunha, R. V., Santana, P. R. \& Carneiro, A. M. (2009). Evidência de Validade entre o Inventário de Percepção de Suporte Familiar - IPSF e Familiograma - FG.

Conselho Federal de Psicologia. (2003). Resolução CFP $n^{o}$ 002/2003. Retrieved March 12, 2008, from http:// www.crpsp.org.br/a_orien/set_projetos_lei.htm

Conte, F. C. S. (2001). Promovendo a relação entre pais e filhos. In M. Deletti (Ed.), Sobre comportamento e cognição (Vol. 2, pp. 159-166). Santo André, SP: ESETec.

D'avila-Bacarji, K. M. G., Maturano, E. M., \& Elias, L. C. S. (2005). Suporte parental: Um estudo sobre crianças com queixas escolares. Psicologia em Estudo, 10, 107-115.

Dundas, I. (1994). The Family Adaptability and Cohesion Scale III in a Norwegian sample. Family Process, 33, 191-202.

Elmaci, F. (2006). The role of social support on depression and adjustment levels of adolescents having broken and unbroken families. Educational Sciences: Theory \& Practice, 6, 421-431.

Feldman, S. S., \& Gehring, T. M. (1988). Changing perceptions of family cohesion and power across adolescence. Child Development, 59, 1034-1045.

Forman, S. G., \& Forman, B. D. (1981). Family environment and its relation to adolescent personality factors. Journal of Personality Assessment, 45, 163-167.

Greenbergher, E., Chen, C., Tally, S. R., \& Dong, Q. (2000). Family peer and individual correlates of depressive sympthomatology among US and Chinese adolescents. Journal of Consulting and Clinical Psychology, 68, 209-219.

Horta, R. L., Horta, B. L., \& Pinheiro, R. T. (2006). Drogas: Famílias que protegem e que expõem adolescentes ao risco. Jornal Brasileiro de Psiquiatria, 55, 268-272.

Ivanova, M. Y., \& Israel, A. C. (2005). Family stability as a protective factor against the influences of pessimistic attributional style on depression. Cognitive therapy and research, 29, 243-251.

Larson, R. W., Richards, M. H., Moneta, G., Holmbeck, G., \& Duckett, E. (1996). Changes in adolescents' daily interactions with their families from ages 10-18: Disengagement and transformation. Developmental Psychology, 32, 744-754.

Montigny, F., Lacharite, C., \& Amyot, E. (2006). The transition to fatherhood: The role of formal and informal structures during the post-partum period. Texto Contexto Enfermagem, 15, 601-609.

Phillips, M. R., West, C. L., Shen, Q., \& Zheng, Y. (1998). Comparison of schizophrenic patients' families and normal families in China, using Chinese version of FACES-II and the Family Environment Scales. Family Process, 37, 95-106.

Ponciano, E. L. T., \& Carneiro, T. F. (2003). Modelos de família e intervenção terapêutica. Interações, 8, 57-80.

Pratta, E. M. M., \& Santos, M. A. (2006). Reflexões sobre as relações entre drogadição, adolescência e família: Um estudo bibliográfico. Estudos de Psicologia, 11, 315-322.

Prieto, G., \& Muñiz, J. (2000). Um modelo para evaluar la calidad de tests usados na Espanha. Retrieved March 25, 2008 from http://www.cop.es/vernumero.asp?id=41

Rigotto, D. M. (2006). Evidências de validade entre suporte familiar, suporte social e autoconceito. Dissertação de Mestrado não-publicada, Universidade São Francisco, Itatiba, SP.

Rocha, M. M., \& Brandão, M. Z. S. (2001). A importância do auto-conhecimento dos pais na análise e modificação de suas interações com os filhos. In M. Deletti (Ed.), Sobre comportamento e cognição (Vol. 2, pp. 133-141). Santo André, SP: ESETEc.
Santos, T. M. M. (2006). Evidência de validade entre percepção de suporte familiar e traços de personalidade. Dissertação de Mestrado não-publicada, Universidade São Francisco, Itatiba, SP.

Souza, M. S. (2007). Suporte familiar e saúde mental: Evidência de validade baseada na relação entre variáveis. Dissertação de Mestrado não-publicada, Universidade São Francisco, Itatiba, SP.

Steinberg, L. (1990). Autonomy, conflict, and harmony in the family relationship. In S. Feldman \& G. R. Elliot (Eds.), At the threshold: The developing adolescent (pp. 255-276). Cambridge, MA: Harvard University Press.

Teodoro, M. L. M. (2005). Kognitive Repräsentationen familiärer Beziehungen._Methodenkritische Untersuchungen zu Kohäsion und Hierarchie innerhalb des familiären Systems. Hamburg, Germany: Verlag Dr. Kovac.

Teodoro, M. L. M. (2006). Afetividade e conflito em díades familiares: Avaliação com o Familiograma. Revista Interamericana de Psicologia, 40, 385-390.

Teodoro, M. L. M., \& Käppler, K. C. (2003). Familiograma: Desenvolvimento de um novo instrumento para a avaliação das relações familiares (Psicopatologia do Desenvolvimento - Relatórios Técnicos). Belo Horizonte, MG: Laboratório de Neuropsicologia do Desenvolvimento, Universidade Federal de Minas Gerais.

Urbina, S. (2007). Fundamentos da testagem psicológica. Porto Alegre, RS: Artmed.

Zdanowicz, N., Janne, P., \& Reynaert, C. (2004). Family, health and adolescence. Psychosomatics, 45, 500-507.
Recebido: 10/06/2008

$I^{a}$ revisão: 08/10/2008

$2^{a}$ revisão: 09/01/2009 Aceite final: 09/01/2009 